\title{
Myeloid Cell Activation Process
}

National Cancer Institute

\section{Source}

National Cancer Institute. Myeloid Cell Activation Process. NCI Thesaurus. Code C40839.

Myeloid Cell Activation involves stimulation of the production and activity of myeloid cells.

Immature myeloid cells sample the surrounding environment for pathogens; when they

contact a presentable antigen they become activated into mature dendritic cells and

migrate to a lymph node where they act as antigen-presenting cells. 